\title{
AN IMPLEMENTATION OF A COMPACT SMART RESISTIVE SENSOR BASED ON A MICROCONTROLLER WITH AN INTERNAL ADC
}

\section{Zbigniew Czaja}

Gdańsk University of Technology, Faculty of Electronics, Telecommunications and Informatics, G. Narutowicza 11/12, 80-233 Gdańsk, Poland (هzbzaja@pg.gda.pl, +4858347 1487)

\begin{abstract}
In the paper a new implementation of a compact smart resistive sensor based on a microcontroller with internal ADCs is proposed and analysed. The solution is based only on a (already existing in the system) microcontroller and a simple sensor interface circuit working as a voltage divider consisting of a reference resistor and a resistive sensor connected in parallel with an interference suppression capacitor. The measurement method is based on stimulation of the sensor interface circuit by a single square voltage pulse and on sampling the resulting voltage on the resistive sensor. The proposed solution is illustrated by a complete application of the compact smart resistive sensor used for temperature measurements, based on an 8-bit ATxmega32A4 microcontroller with a 12-bit ADC and a Pt100 resistive sensor. The results of experimental research confirm that the compact smart resistive sensor has $1^{\circ} \mathrm{C}$ resolution of temperature measurement for the whole range of changes of measured temperatures.
\end{abstract}

Keywords: microcontrollers, resistance measurement, ADC, time-domain measurements.

(C) 2016 Polish Academy of Sciences. All rights reserved

\section{Introduction}

Nowadays, the demand for smart sensors still grows for industrial control and automation, consumer applications, medical applications, military applications, etc. Modern smart sensors integrate analog or digital sensors, conditioning circuits, processing units and communication interfaces. The sensors measure physical variables of monitored environment, objects and processes. The analog sensors provide analog information, which has to be converted to the digital form in conditioning circuits. The digital information is collected, interpreted and used to make decisions by a control unit often based on a microcontroller. The microcontroller is also responsible for sending this information via a communication interface, using a standardized protocol. The smart sensors are often equipped with wireless interfaces what enables using them to build wireless sensor networks.

Resistive sensors are one of the important groups of analog sensors. They are used for monitoring such physical variables, as temperature, ambient humidity, pressure, tension, corrosion [1], and they also can work as gas sensors [2]. Typically, depending on the application, the resistive sensors are arranged in the form of a single-element, a differential configuration or a bridge configuration.

As mentioned earlier, a lot of smart sensors are used in wireless sensor networks. Thus, inter alia, they should meet the criteria of low energy consumption and extremely low cost [3] (in practice: optimally low cost). For these reasons, universal sensor interface chips, e.g. [4, 5], and smart sensor modules based on two-terminal or four-terminal methods, e.g. [6-8], which are too expensive and too complex, are not acceptable for this group of smart sensors.

Therefore, a direct sensor-to-microcontroller approach to passive modulating sensors, especially to resistive sensors, was developed. It is characterized by a low-cost and low-power 
solution of the sensor electronic interface. There are solutions for which resistive sensors [9-12], differential resistive sensors [13] and resistive sensor bridges [14, 15] are directly connected to a microcontroller - without any intermediate active component. Generally, such a direct interface circuit's role is to measure the discharging time of an RC network consisted of resistances of the sensor. The simplicity of the measurement system causes that, unfortunately, the resistance measurement is burdened with a big uncertainty, which results mainly from the parameters of microcontroller pins, predetermined by the manufacturing technology. Obviously, this problem was resolved by extension of the direct sensor-tomicrocontroller interface circuit with additional components, e.g. a calibration resistor [16], MOSFET transistors [17], or also an inverter consisted of two MOSFET transistors [18].

The above methods [9-17] are assumed to use only digital pins and timers/counters of the microcontrollers to determine values of resistive sensors. However, at present almost all modern 8bit and 32-bit microcontrollers are equipped with 10-bit or - more often - 12-bit Analog to Digital Converters (ADCs).

Thus, a new compact smart resistive sensor based only on a microcontroller with an internal $\mathrm{ADC}$ and a direct resistive sensor-to-microcontroller interface is proposed in the paper. This approach does not introduce hardware redundancy to the interface circuit of the smart sensor, because it is based only on hardware already existing in the system (microcontroller pins, its timers/counters and ADCs). It is also characterized by: a simple sensor interface circuit, a short measurement time and good accuracy and resolution, what results in a low-cost, low-power solution of the sensor electronic interface.

Obviously, it is possible to use any digital temperature sensor with a serial interface, what increases the cost, but we should remember that these sensors are characterised by a limited range of chip operating temperature.

The proposed approach will be illustrated on an example of a complete application of the compact smart resistive sensor used for temperature measurements, based on an 8-bit microcontroller and a Pt100 temperature resistive sensor.

\section{Operating principle}

a)

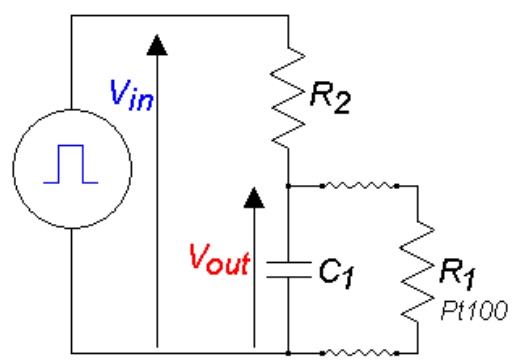

b)

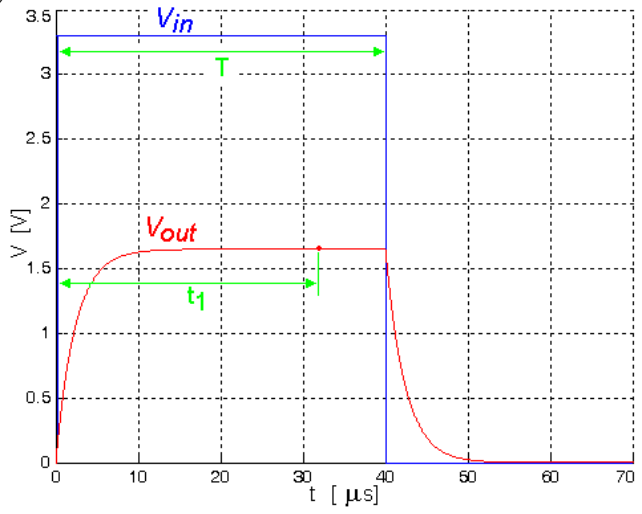

Fig. 1. a) A scheme of the sensor interface circuit; b) timings of the stimulation signal $v_{\text {in }}(t)$ of the sensor interface circuit and its voltage time response $v_{\text {out }}(t)$ for $V_{\text {in }}=3.3 \mathrm{~V}, R_{1}\left(0^{\circ} \mathrm{C}\right)=R_{2}=100 \Omega, C_{1}=43 \mathrm{nF}$.

The proposed direct resistive sensor-to-microcontroller interface for microcontrollers works according to the time-domain measurement method, the general idea of which is presented in [19-21].The method is based on stimulation of a voltage divider (consisting of a reference 
resistor $R_{2}$ working as a current-to-voltage converter, similarly as in [18], and a resistive sensor $R_{1}$ connected in parallel with a capacitor $C_{1}$ (Fig. 1a)) by a single square voltage pulse $v_{i n}$ with an amplitude $V_{\text {in }}$ and a duration time $T$, and on sampling the resulting voltage $v_{\text {out }}$ on the sensor $R_{1}$ at a selected moment $t_{1}$ (Fig. 1b). The main task of the capacitor $C_{1}$ is suppressing interferences, what improves the measurement accuracy and resistance of the interface circuit against interferences and noise, but also extends the measurement time.

\subsection{Circuit analysis}

As shown in Fig. 1a, the sensor interface circuit is a voltage divider, which can be treated as a four-terminal circuit. Hence, its voltage transmittance can be written as:

$$
K(s)=\frac{V_{\text {out }}(s)}{V_{\text {in }}(s)}=\frac{R_{1}}{s\left(R_{1} R_{2} C_{1}\right)+\left(R_{1}+R_{2}\right)} .
$$

Thus, the response signal $V_{\text {out }}(s)$ of the sensor interface circuit is expressed by:

$$
V_{\text {out }}(s)=V_{\text {in }}(s) \frac{R_{1}}{s\left(R_{1} R_{2} C_{1}\right)+\left(R_{1}+R_{2}\right)} .
$$

The sensor interface circuit is stimulated by a single square pulse $v_{i n}(t)$ with the duration time $T$ (Fig. 1b). In the time domain this excitation can be described by the formula:

$$
v_{\text {in }}(t)=V_{\text {in }}(1(t)-1(t-T)) .
$$

The voltage time response $v_{\text {out }}(t)$ is sampled only once, at the moment $t_{1}$. It should be made as quickly as possible to shorten the measurement time and thus to reduce the energy consumption. Therefore, the voltage sampling should be performed during the stimulating pulse. So, it is assumed that the following condition has to be satisfied: $t_{1}<T$. Hence, (3) can be simplified to the form:

$$
v_{\text {in }}(t)=V_{\text {in }}(1(t)) \text { for } t \in(0 ; T) .
$$

Converting it to the Laplace's domain, it is obtained:

$$
V_{\text {in }}(s)=\frac{V_{\text {in }}}{s} .
$$

Finally, the signal $V_{\text {out }}(s)$ is described by:

$$
V_{\text {out }}(s)=\frac{V_{\text {in }} \frac{R_{1}}{R_{1}+R_{2}}}{s\left(s\left(\frac{R_{1} R_{2} C_{1}}{R_{1}+R_{2}}\right)+1\right)}
$$

and - in the time domain - by:

$$
v_{\text {out }}(t)=V_{\text {in }} \cdot \frac{R_{1}}{R_{1}+R_{2}} \cdot\left(1-\exp \left(-t \frac{R_{1}+R_{2}}{R_{1} R_{2} C_{1}}\right)\right) \text { for } t \in(0 ; T) .
$$

As mentioned earlier, the measurement time should be as short as possible. However, the most important aim is to obtain good accuracy and resolution of voltage measurements which takes place at the moment when the greatest possible values of voltages corresponding to given values of the $R_{1}$ resistive sensor (see Fig. 1b) can be measured, i.e., when the capacitor $C_{1}$ is almost fully charged. Obviously, the voltage sampling should be performed as soon as possible 
after charging the capacitor $C_{1}$, and the stimulating pulse should last just as long as to ensure a proper AD conversion. Hence, it is enough to satisfy the following condition:

$$
v_{\text {out }}\left(t_{\infty}\right)-v_{\text {out }}\left(t_{1}\right) \leq V_{L S B},
$$

where $V_{L S B}=\frac{V_{r e f}}{2^{n}}$, and $V_{r e f}$ is the reference voltage of the $n$-bit ADC.

In this case it can be assumed that $v_{\text {out }}\left(t_{1}\right) \approx v_{\text {out }}\left(t_{\infty}\right)$. Hence, $(7)$ can be simplified to the form:

$$
V_{1}=v_{\text {out }}\left(t_{1}\right) \approx V_{\text {in }} \cdot \frac{R_{1}}{R_{1}+R_{2}} .
$$

So, transforming (9), the formula that enables to determine the resistance value of the sensor is:

$$
R_{1}=R_{2} \cdot \frac{V_{1}}{V_{\text {in }}-V_{1}} .
$$

Hence, to determine the resistance value of sensor $R_{1}$ the simple (10) can be used, but in many cases - also in our example illustrating the proposed direct resistive sensor-tomicrocontroller interface for microcontrollers - the values contained in the ADC result registers can directly indicate the measured physical values. For this purpose, a lookup table approach is used, what is presented in the next chapter.

\section{An example of implementation of a compact smart resistive sensor}

In this chapter there are presented the main stages of design of a complete application of the compact smart resistive sensor used for temperature measurements, based on the proposed direct resistive sensor-to-microcontroller interface for microcontrollers with internal ADCs.

\subsection{Selection of component values of the sensor interface circuit}

As mentioned earlier, to illustrate the proposed approach a Pt100 resistive sensor (PT106051 $-100 \Omega$, class B $0.2 \%$, temperature range: from $-50^{\circ} \mathrm{C}$ to $500^{\circ} \mathrm{C}$ ) was chosen and the nominal resistance $R_{1}\left(0^{\circ} \mathrm{C}\right)=100 \Omega$ was assumed. Obviously, the choice may be different - it depends on a given application.

At present, most digital circuits and microcontrollers work at the supply voltage $V_{c c}=3.3 \mathrm{~V}$. Thus, the amplitude $V_{i n}$ of a stimulating pulse was set to the voltage $V_{c c}$.

The resistor $R_{2}$ works as a current limiter, hence its resistance value should be large, but it is a part of the voltage divider (Fig. 1a). Thus, the greater the resistance value of $R_{2}$, the less the voltage $v_{\text {out }}$ at the sensor $R_{1}$, what decreases the measurement accuracy. After simulation experiments it was established that the optimal (and matching the value of $R_{1}$ ) $R_{2}$ value is $100 \Omega$. In this case, the maximum peak value of the current $i(t)$ for $R_{1}\left(-50^{\circ} \mathrm{C}\right)$ is about $41 \mathrm{~mA}$, and after charging the capacitor $C_{1}$ the current value is about $18.3 \mathrm{~mA}$. The minimum level of the current value is $8.7 \mathrm{~mA}$ for $R_{1}\left(500^{\circ} \mathrm{C}\right)$. To stimulate the sensor interface circuit, an inverter built from one IRF7105 component [22] connected to the output pin of the microcontroller is used.

Thanks to this, an adverse impact of electrical parameters of the microcontroller pins on quality of the generated stimulation is eliminated [18].

The capacitor $C_{1}$ is used to suppress interferences and noise. Hence, the larger the capacity value of $C_{1}$, the greater damping of interferences (coming from, inter aria, cables connecting the Pt100 with the rest of the sensor interface circuit (Fig. 1)), but also the longer time of charging the capacitor $C_{1}$, what causes an increased energy consumption by the system. Thus, the choice of the capacitance $C_{1}$ value should be a compromise between effectiveness of 
damping interferences and the duration of the measurement procedure, although - first of all it depends on the design assumptions (e.g. the working environment). For this example it is assumed $C_{1}=43 \mathrm{nF}$.

Summarizing, the nominal values of the sensor interface circuit components are: $R_{1}\left(0^{\circ} \mathrm{C}\right)=$ $R_{2}=100 \Omega, C_{1}=43 \mathrm{nF}$ and $V_{\text {in }}=3.3 \mathrm{~V}$.

\subsection{Determination of the n-bit ADC resolution}

It is assumed that a compact smart resistive sensor for the full range of temperature changes from $\left(-50^{\circ} \mathrm{C}\right.$ to $\left.500^{\circ} \mathrm{C}\right)$ should measure the temperature with $1{ }^{\circ} \mathrm{C}$ resolution, that is with the resolution of the Pt100 resistive sensor defined by the IEC 60751 standard [22].

Thus, a set $\left\{T_{i}\right\}_{i=1, . . I}$, where $I=551$, of integer values of measured temperatures is given, to which a set of resistive values $\left\{R_{1}\left(T_{i}\right)\right\}_{i=1, . . I}$ of the Pt100 resistive sensor [22] is assigned. Hence, for a given temperature value $T_{i}$ the voltage $V_{1}\left(T_{i}\right)$ sampled by the ADC at the moment $t_{1}$ is expressed by:

$$
V_{1}\left(T_{i}\right)=V_{\text {in }} \cdot \frac{R_{1}\left(T_{i}\right)}{R_{1}\left(T_{i}\right)+R_{2}} .
$$

The temperature difference $T_{i+1}-T_{i}=1^{\circ} \mathrm{C}$ corresponds to the voltage difference $\Delta V_{1}\left(T_{i}\right)$ :

$$
\Delta V_{1}\left(T_{i}\right)=V_{1}\left(T_{i+1}\right)-V_{1}\left(T_{i}\right) .
$$

Inserting (11) to (12) gives the following expression:

$$
\Delta V_{1}\left(T_{i}\right)=V_{\text {in }}\left[\frac{R_{1}\left(T_{i+1}\right)}{R_{1}\left(T_{i+1}\right)+R_{2}}-\frac{R_{1}\left(T_{i}\right)}{R_{1}\left(T_{i}\right)+R_{2}}\right],
$$

what finally after transformations gives:

$$
\Delta V_{1}\left(T_{i}\right)=V_{i n}\left[\frac{R_{2}\left(R_{1}\left(T_{i+1}\right)-R_{1}\left(T_{i}\right)\right)}{R_{1}\left(T_{i+1}\right) R_{1}\left(T_{i}\right)+R_{2}\left(R_{1}\left(T_{i+1}\right)+R_{1}\left(T_{i}\right)\right)+R_{2}^{2}}\right] .
$$

To measure the temperature with $1^{\circ} \mathrm{C}$ resolution the voltage difference $\Delta V_{1}\left(T_{i}\right)$ should be less than the voltage $V_{L S B}$ of the $n$-bit ADC (the voltage value for 1 LSB (Least Significant Bit)) defined by the formula:

$$
V_{L S B}(n)=n-\text { bit ADC level }=\frac{V_{r e f}}{2^{n}} .
$$

Figure 2 shows the graph of the voltage difference $\Delta V_{1}\left(T_{i}\right)(14)$ and also voltage levels corresponding to the voltage $V_{L S B}$ values for the 10,12, 14 and 16-bit ADCs (15) for the reference voltage $V_{\text {ref }}=V_{c c}-0.7 \mathrm{~V} \mathrm{[23]} \mathrm{and} V_{i n}=V_{c c}=3.3 \mathrm{~V}$.

It can be seen from Fig. 2 that the 12-bit ADC fully satisfies the previous assumptions. Thus, a microcontroller containing such an ADC should be found. There are a lot of modern 8-bit and 32-bit microcontrollers equipped with a 12-bit ADC.

For the selected compact smart resistive sensor it was decided to choose an 8-bit XMEGA A ATxmega32A4 microcontroller [23, 24]. It has one pipeline 12-bit ADC, which can simultaneously and independently measure voltages at up to four channels, what will be used to improve the measuring accuracy. It also has some interesting peripherals: a 16-bit counter with four-channel Output Compare functions and PWM functions, and an event system 
enabling to change the state of one peripheral to automatically trigger actions in other peripherals, which will be used in the presented application.

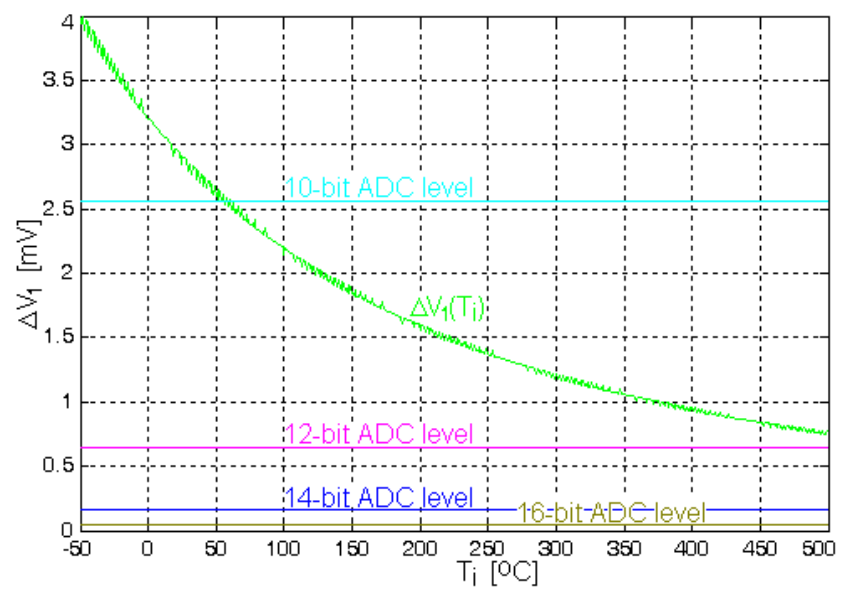

Fig. 2. The graph of the voltage difference $\Delta V_{1}\left(T_{i}\right)$ for the sensor interface circuit for temperatures from $-50^{\circ} \mathrm{C}$ to $500^{\circ} \mathrm{C}, V_{\text {in }}=3.3 \mathrm{~V}, R_{2}=100 \Omega, C_{1}=43 \mathrm{nF}$.

\subsection{Determination of $T$ and $t_{1}$}

The measurement procedure duration determined by the duration $T$ of stimulation, which depends on the sampling moment $t_{1}$, should be possibly short to reduce the energy consumption, but also long enough to ensure the assumed measurement accuracy.

In Subsection 2.1 it is assumed that the time response of the sensor interface circuit at the moment $t_{1}$ has been already stabilized, i.e., after this moment it changes less than $V_{L S B}$ (less than $1 \mathrm{LSB}$ of the ADC). Hence, to determine the formula for $t_{1}$ two formulas (9) and (7) are inserted into the condition (8). After transformations the following is obtained:

$$
t_{1} \geq-\frac{R_{1} R_{2} C_{1}}{R_{1}+R_{2}} \ln \left(\frac{V_{L S B}}{V_{\text {in }}} \frac{R_{1}+R_{2}}{R_{1}}\right) .
$$

The value of the moment $t_{1}$ should be determined for the temperature $T_{501}=500^{\circ} \mathrm{C}$, for which $R_{1}$ has its biggest value $-280.90 \Omega$, that corresponds to the longest time of charging the capacitor $C_{1}$, as shown in Fig. 3. In this case, the condition (8) is fulfilled for the whole range of changes of measured temperatures. For example, for the discussed sensor interface circuit and for $V_{L S B}=635 \mathrm{mV}\left(V_{\text {ref }}=2.6 \mathrm{~V}\right.$ for the 12-bit ADC) the time $t_{1} \geq 25.8 \mathrm{~ms}$.

The duration $T$ of stimulation should be set longer than $t_{1}$ to ensure a proper AD conversion. Hence, it can be written that:

$$
T=t_{1}+t_{A D C_{-} \text {conv }},
$$

where $t_{A D C}$ conv is the time required to carry out the full AD conversion (obviously, performed by the ADC and its supporting software).

For the chosen ATxmega32A4 microcontroller, the 12-bit ADC resolution is possible for the ADC clock frequency $f_{A D C}=500 \mathrm{kHz}$ [24], what gives the sampling time $T_{A D C}=2 \mu \mathrm{s}$. An advantage of the ADC contained in ATxmega32A4 is its ability to simultaneously and independently measure voltages at up to four its channels. Thus, it is a good idea to make use of this hardware feature, what enables using the oversampling technique [25]. 


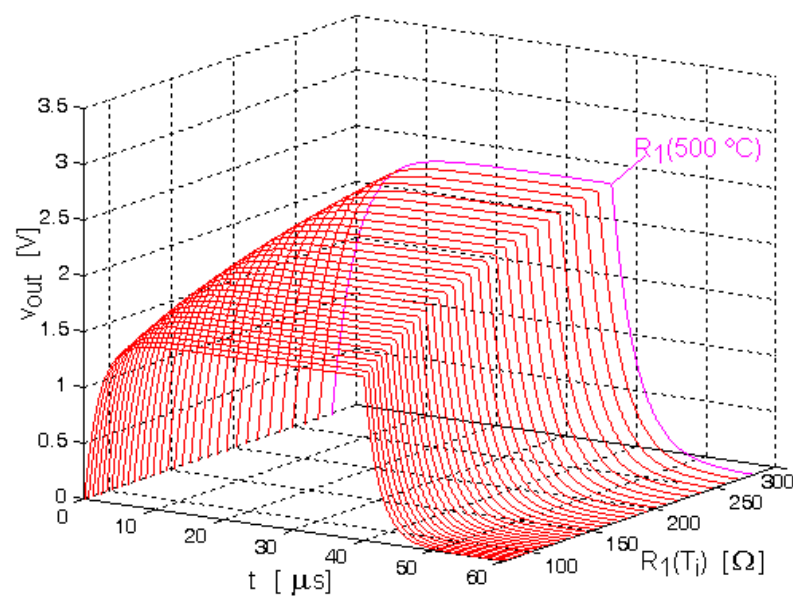

Fig. 3. Timings of the family of time responses of the sensor interface circuit for temperatures from $-50^{\circ} \mathrm{C}$ to $500^{\circ} \mathrm{C}, V_{\text {in }}=3.3 \mathrm{~V}, R_{2}=100 \Omega, C_{1}=43 \mathrm{nF}$.

Hence, the ADC has been configured in such a way, that one trigger signal runs sequentially sampling at four channels connected to the same input with the $v_{\text {out }}$ signal. In this case, averaging the measurement results enables to improve the accuracy of voltage measurements and to reduce the noise variance (or noise power) what, inter alia, influences repeatability of measurements, as confirmed by preliminary examinations. This is done at the expense of extending the AD conversion time: $t_{A D C}$ conv $=4 \cdot T_{A D C}=8 \mathrm{~ms}$. Obviously, this decision is with the constructor of the system. It should be a compromise between the energy consumption of the measurement system and the measurement accuracy.

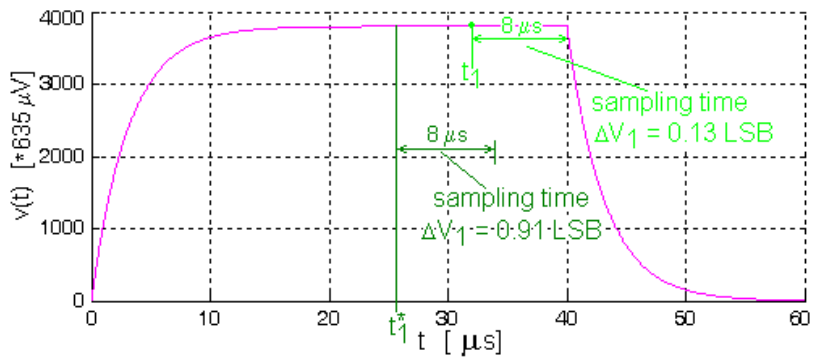

Fig. 4. Timing of the time response of the sensor interface circuit for $R_{1}\left(500^{\circ} \mathrm{C}\right), V_{\text {in }}=3.3 \mathrm{~V}, R_{2}=100 \Omega$, $C_{1}=43 \mathrm{nF}$ with marked ADC conversion times $t_{1}{ }^{*} \approx 25.8 \mu \mathrm{s}$ and $t_{1}=32 \mu \mathrm{s}$.

The prototype compact smart resistive sensor is powered by mains. Thus, achieving the best possible measurement accuracy for a given hardware should be the issue to focus on. For the same reason the excess value $t_{1}=32 \mu \mathrm{s}$ was chosen, for which during $t_{A D C_{-} \text {conv }}=8 \mu \mathrm{s}$ an increase of voltage $v_{1}(t)$ is only $\Delta V_{1}=0.13 \mathrm{LSB}$, i.e., it can be said that this voltage does not change, as shown in Fig. 4, what guarantees correct work of the prototype device during testing the proposed measurement method. For $t_{1}{ }^{*} \approx 25.8 \mu \mathrm{s}$, determined from (16), it is $\Delta V_{1}=0.91 \mathrm{LSB}$. This value is also acceptable and should be used in the final application of a battery-powered compact smart resistive sensor.

Therefore, finally from (17), for $t_{1}=32 \mu$ s the duration $T$ of stimulation is equal to $40 \mu \mathrm{s}$. 


\subsection{The microcontroller application of the compact smart resistive sensor}

The compact smart resistive sensor consists of the ATxmega32A4 microcontroller, the inverter [18] built of two IRF7105 MOSFET transistors [26], and the sensor interface circuit (the resistor $R_{2}$, the PT100 resistive sensor $R_{1}$ and the capacitor $C_{1}$ ), as shown in Fig. 5 . As the communication interface module of the laboratory prototype device, the USART of the microcontroller and the FT232B module (a USB to RS232 converter) of FTDI [27] are used. Obviously, other communication solutions can be used. For instance, if wireless communication is needed, transceivers for IEEE 802.15.4 and ZigBee applications controlled via the microcontroller's SPI can be used.

During the measurement procedure the sensor interface circuit is stimulated by a single square pulse $v_{\text {in }}$ generated on the PC0 pin of the microcontroller and inverted by the MOSFET inverter. The time response $v_{\text {out }}$ of this circuit is sampled four times at the ADC2 pin by the ADC A triggered by the Timer/Counter 0 (TC0) starting at the moment $t_{1}=32 \mu$ s and lasting $8 \mu \mathrm{s}$, and then the stimulation ends.

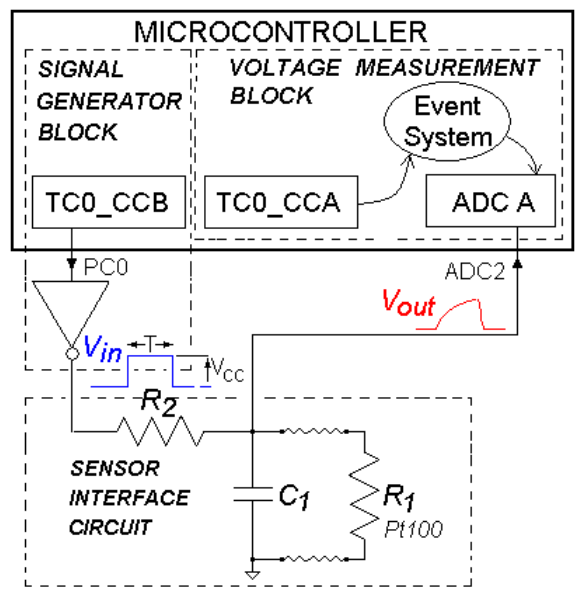

Fig. 5. A block scheme of the compact smart resistive sensor based on the ATxmega32A4 microcontroller.

The duration $T$ of stimulation and the sampling moment $t_{1}$ are counted by only one 16-bit TC0 working in the Normal Mode. It is possible, because the counter has four Compare or Capture (CC) Channels. Each cannel has a 16-bit register TCC0_CCx, where $\mathrm{x}=\mathrm{A}, \mathrm{B}, \mathrm{C}, \mathrm{D}$. The CCx channel continuously compares the counter value (TCC0_CNT) with its TCC0_CCx register. If the counter value is equal to the value written in the TCC0_CCx, the comparator signals a match, and the event and optional interrupt are generated. The channel A (CCA) is used to determine the sampling moment $t_{1}$ (the generated event triggers the AD conversion directly via the event system), and the Channel $B(\mathrm{CCB})$ - to determine the duration $T$ (in the interrupt service generating the stimulation is completed).

All channels $\mathrm{CH} 0, \mathrm{CH} 1, \mathrm{CH} 2$ and $\mathrm{CH} 3$ with their own 16-bit result registers ADCA_CHyRES (where $y=0,1,2,3$ ) of the ADC A are connected to the common ADC2 input pin.

However, an equally important component of the voltage measurement block contained in the microcontroller is the 8-channel event system. Its use simplifies the measurement procedure algorithm and eliminates the problem of program delays. As mentioned earlier, the match signal in the channel CCA of the TC0 is directly transmitted - without participation of the core processor of microcontroller - by the event system, as the event triggers almost simultaneously 
sampling at four channels by the pipelined ADC A (sampling at the next channel is delayed by about one ADC clock cycle $\left.T_{A D C}=2 \mu \mathrm{s}\right)$.

\subsection{The measurement procedure algorithm}

The algorithm of the measurement procedure is partly implemented in the program (Fig. 6a), and partly "included" in the configuration of peripheral devices of the microcontroller forming the measurement microsystem (Fig. 6b): the TC0, the event system, the ADC A. Therefore, it should be noted that the description of the measurement procedure takes into account the simultaneous analysis of the contents of these two figures.

a)

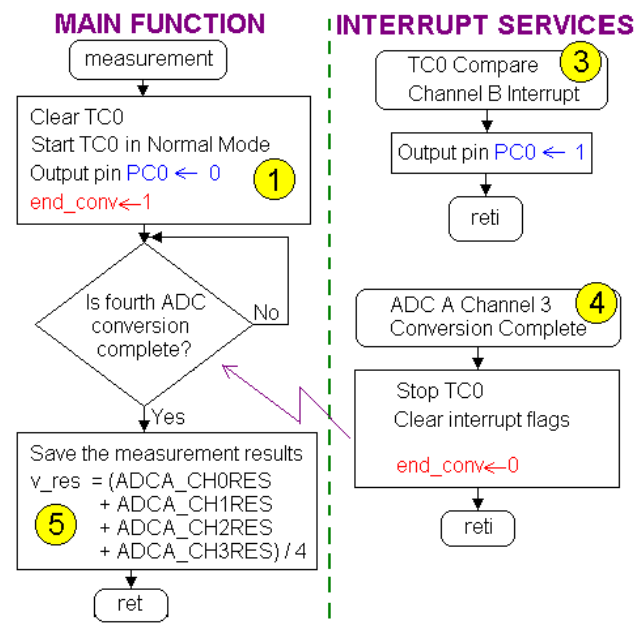

b)

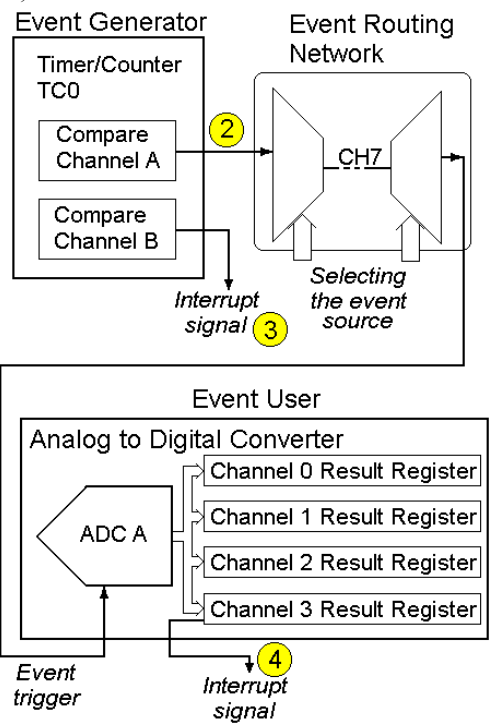

Fig. 6. a) The flowchart of the algorithm of the measurement procedure implemented in the measurement function; b) a block scheme of the measurement microsystem configured from peripheral devices

of the microcontroller ATXmega32A4.

The program performing the measurement procedure consists of three code sections. The code placed in the main body of the measurement function starts the measurement microsystem, and two codes of the interrupt services handle interrupts coming from the TC0 and the ADC A (Fig. 6a). Hence, the following steps of the measurement procedure algorithm (marked on both Fig. 6a and Fig. 6b) can be distinguished:

1. The data register TCC0_CNT of the TC0 is cleared and the TC0 starts in the Normal Mode. At the same time generation of the square pulse starts (the low level is set at the PC0 pin). Also, the flag end_conv is set. It is used for synchronization of software and hardware.

2. The match in the $\bar{C} C A$ channel of the TC0 triggers the AD conversion.

3. The match in the CCB channel of the TC0 generates an interrupt. Its service completes the stimulation (the high level is set at the PC0 pin).

4. Completion of the $\mathrm{AD}$ conversion in the $\mathrm{CH} 3$ (i.e., completion of the last - fourth - $\mathrm{AD}$ conversion) generates an interrupt. During servicing the interrupt the TC0 counter is stopped, all remaining AD conversion complete flags are cleared, the end_conv flag is also cleared causing transition to the main function code. 
5. In this step the four $\mathrm{AD}$ conversion results are averaged and written to the 16-bit $v$ res variable.

\subsection{Determination of the temperature value $T_{i}$}

A lookup table approach is used to determine the temperature value $T_{i}$ directly from the values contained in the $v_{-}$res variable. For this purpose the table of 16-bit variables $V_{I}=$ $\left\{v_{i}\right\}_{i=1, \ldots, I}$ was created and placed in the program memory of microcontroller. The value $v_{i}$ is calculated from the formula:

$$
v_{i}=v_{i, \text { meas }}+\text { round }\left(\frac{v_{i+1, \text { meas }}-v_{i, \text { meas }}}{2}\right) \text {. }
$$

where $v_{i, \text { meas }}$ is the voltage value expressed in the number of bits measured at the moment $t_{1}$ by the ADC A for the $R_{1}\left(T_{i}\right)$ resistance value during testing the compact smart resistive sensor, as described in the next chapter. This value can be also determined through simulation, based on the IEC 60751 standard [22] and (9).

Hence, the value $v_{i, \text { meas }}$ is the middle value of the interval. Thanks to this, a simple conversion algorithm $v_{-}$res $\rightarrow T_{i}$ can be used. The conversion consists in checking whether the condition $v_{\text {res }}<v_{i}$ is satisfied for a given index $i$, starting from $i=0,1, . ., 550$ (starting from 0 is caused by using ANSI C). If this condition is fulfilled for a given value of the index $i$, the algorithm is stopped and the temperature value in Celsius is calculated in the following way: $T_{i}=i-50$.

Thanks to this simple solution of determining the temperature value $T_{i}$, based on the lookup table approach and - also - the simple measurement procedure, 2334 bytes of the program memory $(6.3 \%$ of the program memory of ATxmega32A 4$)$, and 11 bytes of the data memory $(0.3 \%$ of the data memory of ATxmega32A4) are used by the compact smart resistive sensor software written in ANSI C and compiled by AVR8 GCC in Atmel Studio 6. Thus, there is a lot of free space in the program and data memories of the microcontroller for other functionalities, e.g. for communication service functions according to the ISO/IEC/IEEE 21451-001 standard $[28,29]$. In the presented case, the communication is handled via the UART. It additionally occupies only 618 bytes of the program memory.

\section{Experimental results and discussion}

In this chapter the results of experimental research on examining metrological possibilities of the proposed compact smart resistive sensor are presented. A photo of the laboratory compact smart resistive sensor is shown in Fig. 7. It consists of the microcontroller module (the ATXmega32A 4 with the $16 \mathrm{MHz}$ crystal oscillator and other necessary discrete components), the inverter placed on the other side of the printed board, the communication module MMusb232 based on FT232BL and the sensor interface circuit (PT106051, $R_{2}=100 \Omega$, $C_{1}=42.41 \mathrm{nF}-$ the capacitance was measured by the Agilent 4263B LCR METER at $1 \mathrm{kHz}$ ). The voltage values measured by the Agilent 34410A Digital Multimeter are following: $V_{C C}=$ $3.301 \mathrm{~V}$ and $V_{i n}=3.293 \mathrm{~V}$, and the determined reference voltage value is: $V_{\text {ref }}=2.615 \mathrm{~V}$, what gives $V_{L S B}=638.5 \mu \mathrm{V}$.

The measurements were carried out for 551 resistance values of the $R_{1}$ resistor corresponding to all the integer temperature values [22] from the range $-50^{\circ} \mathrm{C}$ to $500^{\circ} \mathrm{C}$ in the laboratory stand. The Pt100 resistive sensor was replaced by a decade TYP DR6a-16 resistor. Hence, the laboratory compact smart resistive sensor was run for each resistance value of the $R_{1}$ resistor physically set in the system. After each measurement the $v \_r e s$ variable was sent to the personal computer via the USB interface. Next, the $v$ res result for a given index $i$ was written in the column $v_{i, \text { meas }}$ of Table 1 . 


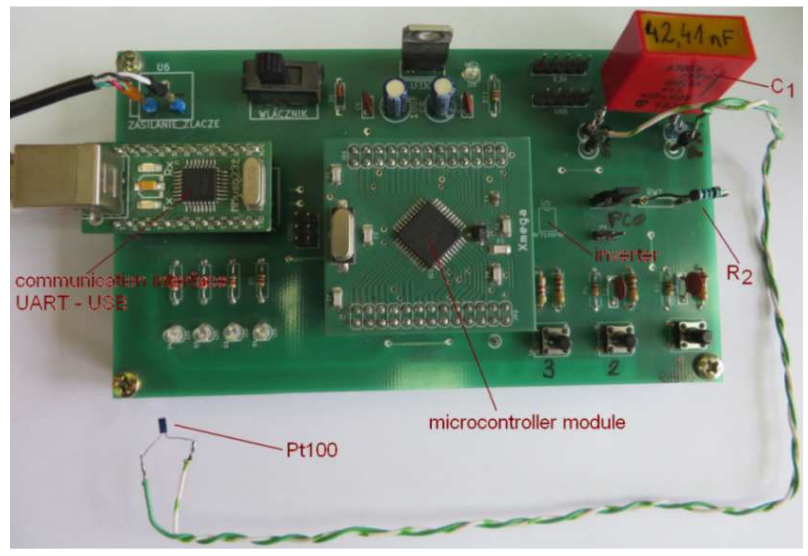

Fig. 7. A photo of the laboratory compact smart resistive sensor.

Since the decade resistor has $0.1 \Omega$ resolution, whereas - according to the IEC 60751 industrial standard [22] - the resistance values of $R_{1}\left(T_{i}\right)$ are defined with $0.01 \Omega$ resolution, the values of $R_{1, i}$ set on the decade resistor had to be rounded in the following way:

$$
R_{1, i}=\operatorname{round}\left(R_{1}\left(T_{i}\right) \cdot 10\right) / 10 \text {. }
$$

Table 1 presents only a chosen fragment of the measurement results obtained during examinations. All 501 measurement results are presented in the graphical form in Fig. 8.

Table 1. A fragment of measurement results made during examinations of the compact smart resistive sensor.

\begin{tabular}{|r|c|c|c|}
\hline $\boldsymbol{i}$ & $\boldsymbol{T}_{\boldsymbol{i}}\left[{ }^{\circ} \mathbf{C}\right]$ & $\mathrm{Pt} \mathbf{1 0 0}-\boldsymbol{R}_{\mathbf{1}, \boldsymbol{i}}[\boldsymbol{\Omega}]$ & $\left.\boldsymbol{V}_{\boldsymbol{i} \text {,meas }}{ }^{*}{ }^{\mathbf{6} 38.5} \boldsymbol{\mu} \mathbf{V}\right]$ \\
\hline 0 & -50 & 80.3 & 2314 \\
\hline 1 & -49 & 80.7 & 2320 \\
\hline 2 & -48 & 81.1 & 2326 \\
\hline 3 & -47 & 81.5 & 2332 \\
\hline 4 & -46 & 81.9 & 2337 \\
\hline 5 & -45 & 82.3 & 2343 \\
\hline 6 & -44 & 82.7 & 2349 \\
\hline 7 & -43 & 83.1 & 2354 \\
\hline 8 & -42 & 83.5 & 2360 \\
\hline$\ldots$ & $\ldots$ & $\ldots$ & $\ldots$ \\
\hline 548 & 498 & 280.3 & 3715 \\
\hline 549 & 499 & 280.6 & 3716 \\
\hline 550 & 500 & 281.0 & 3717 \\
\hline
\end{tabular}

The experimental research shows that the proposed compact smart resistive sensor based on the new direct resistive sensor-to-microcontroller interface for microcontrollers with internal ADCs works correctly in the whole established range of temperatures measured by the Pt100 resistive sensor. The temperature resolution for this range is at least at the level of $1^{\circ} \mathrm{C}$. For low temperatures it is even possible to obtain a higher resolution (see Table 1), but the $1^{\circ} \mathrm{C}$ resolution is used here, according to the IEC 60751 industrial standard [22]. Furthermore, thanks to four times oversampling and the use of $C_{1}$ capacitor, the measurement results featured high 
repeatability, i.e., a good resistance against interferences occurring during measurements in the laboratory stand.

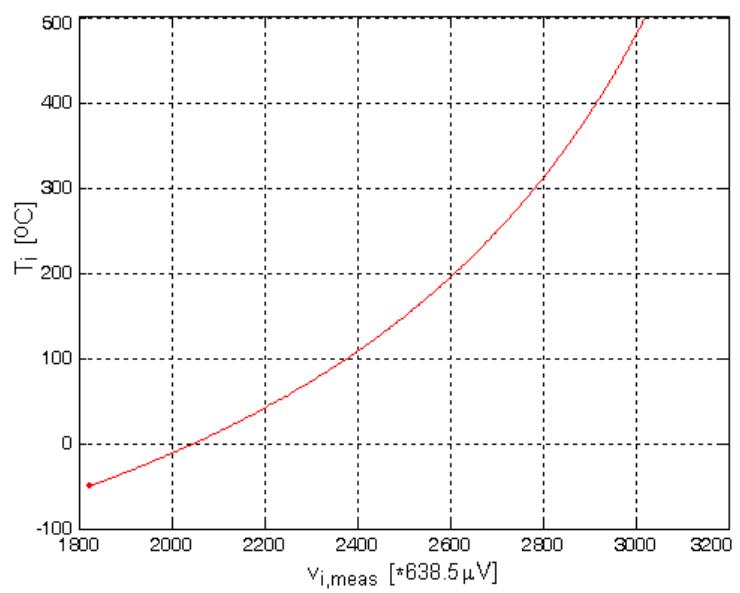

Fig. 8. The graph of temperature values $T_{i}$ in function of voltages measured by the microcontroller ADC for the compact smart resistive sensor.

As mentioned earlier, the measured values $\left\{v_{i, \text { meas }}\right\}_{i=1, . ., I}$ were used to create the lookup table for the conversion algorithm $v_{-}$res $\rightarrow T_{i}$. Hence, an advantage of this approach is the fact that this table already contains experimentally calibrated data.

\section{Conclusions}

In the paper a new compact smart resistive sensor solution based on a direct resistive sensorto-microcontroller interface for microcontrollers with internal ADCs and a new approach to determining resistive sensor parameters using a single square pulse and the time domain method developed for this interface system are presented.

An example of complete application of the compact smart resistive sensor for temperature measurements based on this system for the Pt100 temperature resistive sensor and the 8-bit ATXmega32A4 microcontroller was also presented. All stages of design of the compact smart resistive sensor and the results of experimental research of this device were presented.

Summarizing, thanks to the proposed approach based on the direct resistive sensor-tomicrocontroller interface for microcontrollers with internal ADCs, the compact smart resistive sensors based on 8-bit microcontrollers and simple sensor interface circuits can be built. Hence, the approach enables to obtain a low-cost and low-power solution of the sensor electronic interfaces. It follows from the fact that the proposed simple measurement procedure needs a short measurement time, and determination of the temperature value $T_{i}$ does not need any calculation, because it is based on the lookup table approach. Thanks to using the 12-bit internal $\mathrm{ADC}$ of microcontroller, the $1^{\circ} \mathrm{C}$ resolution of temperature measurement for the whole range of changes of temperatures measured by the compact smart resistive sensor was obtained, which was confirmed by experiments. Additionally, the occupation of program and data memories by the compact smart resistive sensor software is small, what enables extending the functionality of this device, e.g. adding communication service functions.

These advantages, especially a low cost and a low power consumption, give possibilities of using the compact smart resistive sensors in both battery-powered and wireless sensor network applications, e.g. basing on the ZigBee protocol. 


\section{References}

[1] Smulko, J., Darowicki, K., Wysocki, P. (1998). Digital measurement system for electrochemical noise. Polish Journal of Chemistry, 72(7), 1237-1241.

[2] Kotarski, M., Smulko, J. (2009). Noise measurement set-ups for fluctuations-enhanced gas sensing. Metrol. Meas. Syst., 16(3), 457-464.

[3] Kuorilehto, M., Kohvakka, M., Suhonen, J., Hamalainen, P., Hannikainen, M., Hamalainen, T.D. (2007). Ultra-low energy wireless sensor networks in practice. John Wiley \& Sons, Ltd. Great Britain.

[4] Yurish, S.Y. (2010). Universal Interfacing Circuit for Resistive-Bridge Sensors. First International Conference on Sensor Device Technologies and Applications., Venice, Italy, 211-216.

[5] Falconi, C., Martinelli, E., Di Natale, C., D'Amico, A., Maloberti, F., Malcovati, P., Baschirotto, A., Stornelli, V., Ferri, G. (2007). Electronic interfaces. Sensors and Actuators B, (121), 295-329.

[6] PT100 RTD Evaluation Board User's Guide. Datasheet for Microchip Technology Inc. 2007. http://ww1.microchip.com/downloads /en/DeviceDoc/51607b.pdf

[7] Positive Analog Feedback Compensates PT100 Transducer. Datasheet for Maxim Integrated Products Inc. 2005. http://www.maximintegrated.com/en/app-notes/index.mvp/id/3450

[8] AN-1559 Practical RTD Interface Solutions. Datasheet for Texas Instruments. 2013. http://www.ti.com/lit/an/snoa481b/snoa481b.pdf

[9] Reverter, F., Jordana, J., Gasulla, M., Pallàs-Areny, R. (2005). Accuracy and resolution of direct resistive sensor-to-microcontroller interfaces. Sensors and Actuators A, (121), 78-87.

[10] Bengtsson, L. (2012). Direct analog-to-microcontroller interfacing. Sensors \& Actuators A, (179), 105- 113.

[11] Reverter, F., Gasulla, M., Pallàs-Areny, R. (2007), Analysis of power-supply interference effects on direct sensor-to-microcontroller interfaces. IEEE Transactions on Instrumentation and Measurement, 1(56), $171-177$.

[12] Reverter, F. (2012). The Art of Directly Interfacing Sensors to Microcontrollers. Journal of Low Power Electronics and Applications, (2), 265-281.

[13] Reverter, F., Casas, Ò. (2009). Interfacing differential resistive sensors to microcontrollers: A direct approach. IEEE Transactions on Instrumentation and Measurement, 10(58), 3405-3410.

[14] Sifuentes, E., Casas, O., Reverter, F., Pallàs-Areny, R. (2008). Direct interface circuit to linearise resistive sensor bridges. Sensors and Actuators A, (147), 210-215.

[15] Jordana, J., Pallàs-Areny, R. (2006). A simple, efficient interface circuit for piezoresistive pressure sensors. Sensors \& Actuators A, (127), 69-73.

[16] Kokolanski, Z., Gavrovski, C., Dimcev, V. (2014). Modified single point calibration with improved accuracy in direct sensor-to-microcontroller interface. Measurement, (53), 22-29.

[17] Kokolanski, Z., Gavrovski, C., Dimcev, V., Makraduli, M. (2013). Hardware techniques for improving the calibration performance of direct resistive sensor-to-microcontroller interface. Metrol. Meas. Syst., 4(20), $529-542$.

[18] Czaja, Z. A microcontroller system for measurement of three independent components in impedance sensors using a single square pulse. Sensors \& Actuators A, 173, 284-292.

[19] Czaja, Z. (2009). A method of fault diagnosis of analog parts of electronic embedded systems with tolerances. Measurement, (42), 903-915.

[20] Czaja, Z. (2008). Using a square-wave signal for fault diagnosis of analog parts of mixed-signal electronic embedded systems. IEEE Transactions on Instrumentation and Measurement, (57), 1589-1595.

[21] Toczek, W., Czaja, Z. (2011). Diagnosis of fully differential circuits based on a fault dictionary implemented in the microcontroller systems. Microelectronics Reliability, 8(51), 1413-1421.

[22] International Standard, IEC 60751:2008. (2008). Industrial platinum resistance thermometers and platinum temperature sensors, 2.0 edn. International Electrotechnical Commission. Geneva, Switzerland. 
[23] 8/16-bit AVR XMEGA A4 Microcontroller. ATxmega128A4, ATxmega64A4, ATxmega32A4, ATxmega16A4. Datasheet for Atmel Corporation. 2013. http://www.atmel.com/Images/Atmel-8069-8-and16-bit-AVR-AMEGA-A4-Microcontrollers_Datasheet.pdf

[24] 8-bit XMEGA A Microcontroller, XMEGA AU MANUAL. Datasheet for Atmel Corporation. 2013. http://www.atmel.com/images/atmel-8331-8-and-16-bit-avr-microcontroller-xmega-au_manual.pdf

[25] Watkinson, J. (2001). The art of digital audio. 3rd ed., Elsevier.

[26] IRF7105 HEXFET Power MOSFET. Datasheet for International Rectifier. 2003. http://www.irf.com/product-info/datasheets/data/irf7105.pdf

[27] FT232BL /BQ USB UART IC Datasheet, Version 2.2. Datasheet for Future Technology Devices International Limited. 2011. http://www.ftdichip.com/Support/Documents/DataSheets/ICs/DS_FT232BL BQ

[28] Liu, Z., Banakhr, F., Monte, G., Huang, V. (2015). Using Algorithms on Smart Transducer: An IEEE Standard Perspective. IEEE Sensors Journal, 15(5), 2523-2530.

[29] Giorgi, G. (2015). ISO/IEC/IEEE 21451 Compliant Sensor Nodes for Energy-Aware Wireless Sensor Networks. IEEE Sensors Journal, 15(5), 2488-2496. 